\title{
Multisensory Activation of the Intraparietal Area When Classifying Grating Orientation: A Functional Magnetic Resonance Imaging Study
}

\author{
Ryo Kitada, ${ }^{1}$ Tomonori Kito, ${ }^{2}$ Daisuke N. Saito, ${ }^{3,4}$ Takanori Kochiyama, ${ }^{5}$ Michikazu Matsumura, ${ }^{6}$ Norihiro Sadato, ${ }^{3,4,7}$ \\ and Susan J. Lederman ${ }^{1}$ \\ ${ }^{1}$ Department of Psychology, Queen's University, Kingston, Ontario, Canada K7L 3N6, ${ }^{2}$ Graduate School of Health and Sports Science, Juntendo University, \\ Inba 270-1606, Japan, ${ }^{3}$ National Institute for Physiological Sciences, Okazaki 444-8585, Japan, ${ }^{4}$ Japan Science and Technology Corporation/Research \\ Institute of Science and Technology for Society, Kawaguchi 332-0012, Japan, ${ }^{5}$ Faculty of Engineering, Kagawa University, Takamatsu 761-0396, Japan, \\ ${ }^{6}$ Graduate School of Human and Environmental Studies, Kyoto University, Kyoto 606-8501, Japan, and 7Department of Functional Neuroimaging, Faculty \\ of Medical Sciences, University of Fukui, Fukui 910-1193, Japan
}

Humans can judge grating orientation by touch. Previous studies indicate that the extrastriate cortex is involved in tactile orientation judgments, suggesting that this area is related to visual imagery. However, it has been unclear which neural mechanisms are crucial for the tactile processing of orientation, because visual imagery is not always required for tactile spatial tasks. We expect that such neural mechanisms involve multisensory areas, because our perception of space is highly integrated across modalities. The current study uses functional magnetic resonance imaging during the classification of grating orientations to evaluate the neural substrates responsible for the multisensory spatial processing of orientation. We hypothesized that a region within the intraparietal sulcus (IPS) would be engaged in orientation processing, regardless of the sensory modality. Sixteen human subjects classified the orientations of passively touched gratings and performed two control tasks with both the right and left hands. Tactile orientation classification activated regions around the right postcentral sulcus and IPS, regardless of the hand used, when contrasted with roughness classification of the same stimuli. Right-lateralized activation was confirmed in these regions by evaluating the hemispheric effects of tactile spatial processing with both hands. In contrast, visual orientation classification activated the left middle occipital gyrus when contrasted with color classification of the same stimuli. Furthermore, visual orientation classification activated a part of the right IPS that was also activated by the tactile orientation task. Thus, we suggest that a part of the right IPS is engaged in the multisensory spatial processing of grating orientation.

Key words: discrimination; fMRI; multisensory; somatosensory; spatial reference frame; vision

\section{Introduction}

There has been considerable interest in the neural mechanisms involved in judging tactile orientation, because this task is considered a valid indicator of tactile spatial acuity (Van Boven and Johnson, 1994a,b). The tactile system is less efficient than the visual system in both speed and accuracy when processing the spatial attributes of objects (Jones and Lederman, 2006). One possible heuristic for tactile spatial processing is that tactile inputs are translated into a corresponding visual representation

Received Nov. 1, 2005; revised April 19, 2006; accepted June 5, 2006.

This work was supported by Grant-in-Aid for Scientific Research \$\#17100003 from the Japan Society for the Promotion of Science to N.S., by Grant-in-Aid for Scientific Research $018 \# 17021045$ from the Japanese Ministry of Education, Culture, Sports, Science, and Technology to N.S., and by grants from the National Sciences and Engineering Research Council of Canada and the Canadian Institutes of Health Research to S.J.L. We thank M. Shimura for his kind cooperation in crafting the surfaces of linear gratings; Y. Nawa, K. Yamanaka, Dr. H. Mochiyama, and M. Murase for their technical support; and T. Takei and Dr. A. Sano for their cooperation in recruiting subjects.

Correspondence should be addressed to Dr. Norihiro Sadato, Department of Cerebral Research, National Institute for Physiological Sciences, 0kazaki, Aichi 444-8585, Japan. E-mail: sadato@nips.ac.jp.

DOI:10.1523/JNEUROSCI.0822-06.2006

Copyright $\odot 2006$ Society for Neuroscience $\quad$ 0270-6474/06/267491-11\$15.00/0 that is further processed by the visual system (visual mediation heuristics) (Lederman et al., 1990). The extrastriate cortex, near the parieto-occipital fissure, is activated during the tactile discrimination of grating orientations (Sathian et al., 1997; Zangaladze et al., 1999). Because this activation is associated with subjects reporting the use of visual imagery, it has been proposed that this area is engaged in the visual mediation heuristic of gratings.

However, it is still unclear which neural mechanisms are crucial for processing tactile orientation, because visual mediation is not necessarily required for all tactile spatial tasks (Marmor and Zaback, 1976; Carpenter and Eisenberg, 1978). One possible mechanism might involve not only sensory-specific areas including the postcentral gyrus, but also multisensory areas, because our spatial perception of orientation is highly integrated. For instance, the anterior part of the intraparietal sulcus (IPS) was activated during the passive tactile discrimination of the shape of ellipsoids compared with a rest condition in a positron emission tomography (PET) study (Bodegard et al., 2001). This area was also active during tactile grating orientation discrimination relative to judging the spacing between gratings in a functional mag- 
netic resonance imaging (fMRI) study (Zhang et al., 2005). In contrast, part of the IPS was activated during the visual discrimination of grating orientation compared with visual detection of the same stimulus in a PET study (Vandenberghe et al., 1996) and the detection of the dimming of the fixation point in an fMRI study (Faillenot et al., 2001). These findings indicate that a subregion within the human IPS might construct a multisensory representation of orientation. However, there is, as yet, little evidence for the existence of such a multisensory IPS subregion.

In the present study, we used fMRI to test the hypothesis that a region within the IPS is engaged in multisensory spatial processing during the classification of grating orientations. To visualize the neural substrates of its spatial processing, the orientation task for each sensory modality was contrasted with its own control condition (roughness classification for touch, color classification for vision). The premise is that the orientation classification relies on some form of spatial reference system as a reference, whereas the control tasks do not. Each control condition was designed to control for the sensory input, responses, and task demands within one sensory modality, that is, touch or vision. To assess the existence of a multisensory subregion for orientation classification, we examined the activity of the IPS within the regions that were activated during the tactile orientation task during a visual orientation task.

\section{Materials and Methods \\ Subjects}

Sixteen healthy Japanese volunteers (10 males and 6 females) aged 22-47 years, including students and researchers, participated in the fMRI study. All participants were right-handed according to the Edinburgh handedness inventory (Oldfield, 1971). All subjects gave informed written consent, and the study was approved by the ethical committee of the $\mathrm{Na}$ tional Institute for Physiological Sciences of Japan. None of the volunteers had a history of symptoms requiring neurological, psychological, or other medical care.

\section{Tactile stimulus}

The rectangular linear gratings were prepared from plastic sheets with a photosensitive layer (Makoto Craft, Yokohama, Japan). The height and width of the ridges were constant at 1.0 and $0.5 \mathrm{~mm}$, respectively. The length and width of the gratings were 20 and $\sim 40 \mathrm{~mm}$, respectively. We used nine different gratings, each of which was produced with one of three different orientations $\left(-30,0\right.$, and $\left.30^{\circ}\right)$ (Fig. $1 B$ ) and one of three different degrees of roughness. Three gratings at each orientation contained different
A Perceived roughness magnitude of oriented gratings

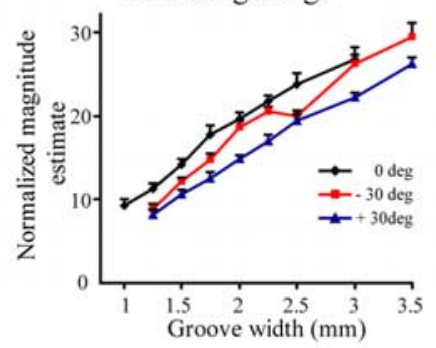

C Stimulus application

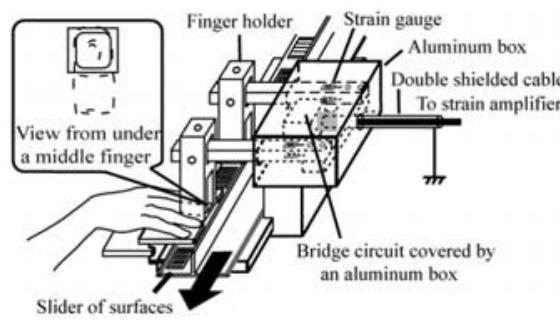

B Stimuli used for the tactile fMRI study

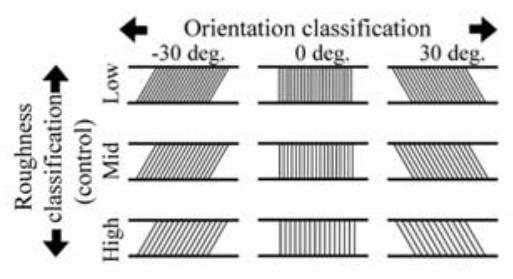

D Task schedule of each hand

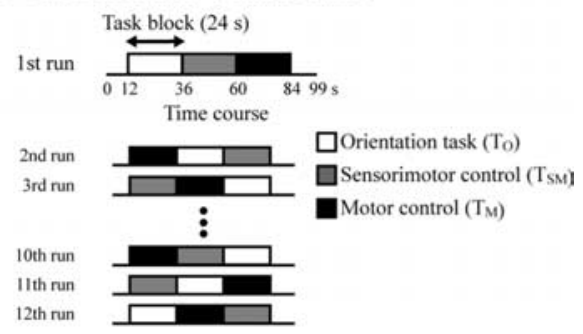

E Task block

Orientation task $\left(\mathrm{T}_{\mathrm{O}}\right)$

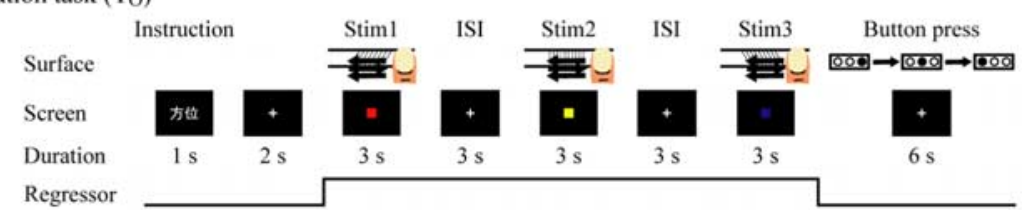

Sensorimotor control (Roughness task, $\mathrm{T}_{\mathrm{SM}}$ )

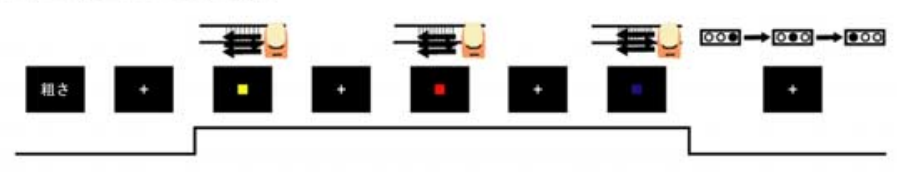

Motor control $\left(\mathrm{T}_{\mathrm{M}}\right)$

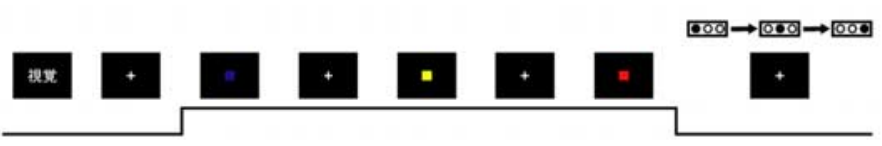

The other regressors

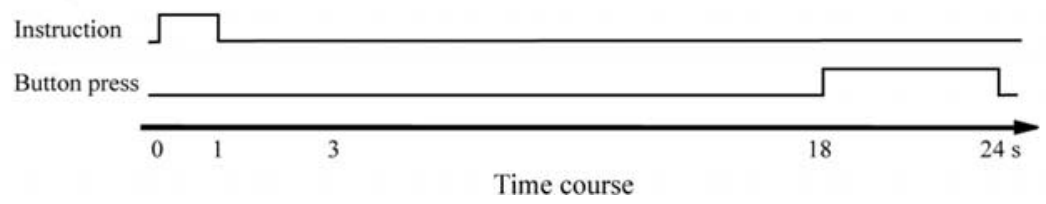

Figure 1. The tactile tasks. A, Roughness-magnitude estimation of gratings. The two pilot psychophysical experiments were conducted to equate the magnitude of perceived roughness between the different orientations. In the conventional magnitudeestimation experiment, the oriented gratings $\left( \pm 30^{\circ}\right)$ were perceived as smoother than the $0^{\circ}$ gratings of the same groove width. $B$, Tactile stimuli. In the fMRI study, we used nine different grating surfaces, each of which had one of three different orientations $\left(-30,0\right.$, and $\left.+30^{\circ}\right)$ and one of three different degrees of roughness. C, Apparatus for stimulation. The subjects placed their middle fingertips lightly on the surface of a slider through a bore in the plastic holder; the other fingers rested on a supporting plastic frame just above the surface. Vertical pressure was monitored by a strain gauge during the experiment. $\boldsymbol{D}$, Task schedule. Three classification tasks were performed in a run. The order of the tasks was pseudorandomized. The subjects performed 12 runs for each hand. This procedure was repeated for both hands. Baseline periods were added before the first task and after the third task block. $\boldsymbol{E}$, Task block. Each run consisted of three $24 \mathrm{~s}$ task blocks. The subjects fixated on the visual cues on the screen. The $3 \mathrm{~s}$ tactile stimulation (Stim) was alternated with a 3 s interstimulus interval (ISI). The subjects judged the grating orientation during the orientation task $\left(T_{0}\right)$, the roughness of the gratings during the sensorimotor control condition $\left(T_{S M}\right)$, and the color of the squares on the screen during the motor control condition $\left(T_{M}\right)$. The color of the squares was pseudorandomly chosen, independently of the orientation or roughness of the gratings. After the stimulation with the third surface, the subjects were asked to press three buttons in succession with the fingers of their other hand. The three buttons were aligned orthogonal to the body axis. The neural activity during the instruction periods, response periods, and three test periods were each modeled with boxcar functions. The regressors shown in the figure were convolved with a canonical hemodynamic-response function. A single task block was immediately followed by the subsequent block of another task. 
groove widths $\left(\sim 1.50,2.00\right.$, and $2.50 \mathrm{~mm}$ for $-30^{\circ} ; \sim 1.25,1.75$, and 2.25 $\mathrm{mm}$ for $0^{\circ}$; and $\sim 1.75,2.25$, and $3.00 \mathrm{~mm}$ for $30^{\circ}$ ).

These different groove widths were specified for each orientation to match the perceived magnitude of roughness between the orientations. The choice of groove widths was based on two psychophysical pilot experiments, which were conducted outside of the MR scanner. In the first experiment, we obtained psychophysical roughness functions for each of the three grating orientations using a conventional magnitudeestimation procedure. Twelve right-handed subjects participated in this experiment. Tactile stimulation of the right middle fingertip was performed using 24 gratings (eight surfaces for each orientation) (Fig. $1 \mathrm{~A}$ ). Each of the gratings was swept under the right middle finger three times. The subjects were asked to estimate the roughness magnitude of each grating. Each surface was presented three times. The order in which the surfaces were presented was pseudorandomized. The mean force was controlled at $\sim 30 \mathrm{~g}$ by a balance, and the average speed was controlled by the autostimulator at $\sim 50 \mathrm{~mm} / \mathrm{s}$. The other procedures, and the method of analysis, were described previously (Lederman and Taylor, 1972; Lederman, 1981). The psychophysical roughness functions were then used to select eight pairs $\left( \pm 30^{\circ}\right)$ of groove-width values that matched the perceived roughness magnitudes of the eight $0^{\circ}$ gratings. Five subjects chose a pair of gratings with $\pm 30^{\circ}$ orientations to achieve the closest match in perceived roughness magnitude with each of the $0^{\circ}$ gratings. We chose the groove width that was selected most frequently across all subjects.

In the second pilot experiment, we chose three $0^{\circ}$ gratings of eight, such that the difference in groove width was equivalent between the grating pairs. Using the nine surfaces (three $0^{\circ}$ gratings with three pairs of $\pm 30^{\circ}$ ), we confirmed that performance accuracy was matched between the orientation and roughness classification tasks.

\section{Tactile stimulus application}

Two sets of three gratings (6 surfaces) were glued onto an L-shaped slider. One set of three gratings had the same magnitude of perceived roughness with three different orientations. The other set of gratings comprised gratings of the same orientation but with three different levels of roughness. The first set was used for the tactile orientation task, and the second set was used for the tactile sensorimotor (roughness) control task. Eighteen sliders were prepared (three surfaces $\times$ two sets $\times 18$ sliders $=108$ surfaces altogether). Twelve sliders were pseudorandomly chosen for each hand for each subject, so that the tactile orientation task and its sensorimotor control contained identical surfaces. A single slider was used with each run. The order of surfaces in each set and the order of the two different sets on a slider were pseudorandomized.

The subjects lay supine on a bed with their eyes open and their ears plugged and were instructed to relax. The subjects were asked to fixate on a white cross (viewing angle, $1.7 \times 1.7^{\circ}$ ) on a semitransparent viewing screen, projected from an LCD projector (DLA-M200L; Victor, Yokohama, Japan) through a mirror. The subjects' right arms were extended along the sides of their body and comfortably supported by a cushion. The subjects placed their right middle fingertips lightly on the surface of the slider through a bore in the plastic holder, with the other fingers resting on a plastic frame just above the surface (Fig. 1C). The distalproximal axis of the middle finger was parallel to the body axis of the subjects. The bore size was $18 \mathrm{~mm}$ in the proximal-distal direction and $17 \mathrm{~mm}$ in the lateral-medial direction. The thickness of the plastic holder was $2 \mathrm{~mm}$. The finger was immobilized against the horizontal movement of the slider. We explained to the subjects that they should avoid applying excessive pressure to the stimulator. The experimenter did not observe any conspicuous movement by any subject when presenting the stimuli to the fingertip. The vertical pressure was also monitored by a strain gauge during the experiment. The subjects' left hands were extended along the side of their body and placed on a response box. The index, middle, and ring fingers of the left hand were placed on each of three buttons of the response box. The three buttons were aligned orthogonal to the body axis. The same procedure was followed when the left middle finger was stimulated, except that the stimulated and response hands were interchanged.

An experimenter moved the slider back and forth in the horizontal direction, guided by auditory cues. These cues were presented only to the experimenter through a pair of ceramic-condenser headphones (Hitachi Medical Corporation, Tokyo, Japan). The rail moved quietly, without making any sound that could be related to the task. The range of surface displacement was $\sim 50 \mathrm{~mm}$ and was demarcated with black ink on each stimulus plate. The mean speed of the slider was $\sim 50 \mathrm{~mm} / \mathrm{s}$ (HT-5100; Ono Sokki, Yokohama, Japan).

\section{Tactile task}

The subjects first performed the three tactile tasks (orientation, its sensorimotor control, and motor control) and then performed the two visual tasks (orientation, its sensorimotor control). The tactile tasks were designed to show the cortical regions related to tactile-orientation classification by contrasting it with its own control condition. This contrast was designed to subtract the activity related to factors including the sensory input, task demand, and responses.

Both right and left hands were tested in the tactile tasks. The order of the two hand conditions was counterbalanced within the group of subjects. A single run consisted of three 24 s blocks. In a single block, the subjects were engaged in one of the three tasks. The subjects went through 12 runs for each hand (Fig. 1D). The order of the tasks was pseudorandomized in each run. Before scanning, in the MRI room, the subjects practiced the tactile classification tasks using the same sets of gratings until they reached a specified level of performance of at least seven correct judgments of nine surfaces.

Tactile orientation task $\left(T_{O}\right)$. The subjects were instructed to classify the orientation of the linear gratings under their middle finger while fixating the visual cues on the screen (Fig. $1 E$, top). A single task block consisted of the instruction ( $3 \mathrm{~s})$, test (15 s), and response ( $6 \mathrm{~s})$ periods. The instruction cue (viewing angle, $3.6 \times 6.4^{\circ}$ ) was presented in Chinese characters for the first second of the instruction period. Then, the finger pads were stimulated by the three surfaces during the test period. For each surface, the slider was moved three times in $3 \mathrm{~s}: 50 \mathrm{~mm}$ in the left-to-right direction for the first second, $50 \mathrm{~mm}$ in the right-to-left direction for the next second, and $50 \mathrm{~mm}$ in the left-to-right direction for the final second. Three seconds of tactile stimulation alternated with a $3 \mathrm{~s}$ interstimulus interval. The surface always moved from the smooth portions beside the gratings. During stimulation with each surface, a square of one of three different colors (blue, yellow, and red) was presented on the center of the screen (viewing angle, $1.7 \times 1.7^{\circ}$ ). The order of the colors was pseudorandomized to avoid any possible association between a given color and a certain grating orientation. After the presentation of the third surface, the subjects were asked to press three buttons in succession, which were assigned to three different orientations. The subjects pushed the left button for left-oriented $\left(-30^{\circ}\right)$ gratings, the middle button for $0^{\circ}$ gratings, and the right button for right-oriented $\left(+30^{\circ}\right)$ gratings. The subjects were asked to press all buttons accurately within $6 \mathrm{~s}$. Eight functional volumes ( $24 \mathrm{~s} ; 3 \mathrm{~s}$ per volume) were acquired in each block.

Sensorimotor control (roughness task, $T_{S M}$ ). The roughness classification of the grating surface was chosen as a tactile control condition. The premise was that the spatial processing of orientation relies on some form of spatial reference system, whereas roughness classification does not require such a stage of processing. Rather, roughness classification depends on comparison of the perceived roughness magnitude of the current surface to those of the other stimulus surfaces (Lederman and Klatzky, 1997). This condition was designed to control for the sensory input factors, as well as the response and task demands of the orientation task. The experimental design was the same as in the orientation task, except for the arrangement of the stimuli and the task instructions. The surfaces for this task possessed the same orientations with three different levels of roughness in each run. The subjects were instructed to classify the roughness of the linear gratings (Fig. $1 E$, middle). The order of the colors of the squares was also pseudorandomized to avoid any associations between a given color and a certain roughness of gratings. The subjects pushed the left button for the smoothest gratings, the middle button for intermediately rough gratings, and the right button for the roughest gratings.

Motor control. This condition was designed to control for visual input 
and the responses during the orientation task. In this condition, only visual (color) cues were presented on the screen, and there was no tactile stimulation (Fig. $1 E$, bottom). The subjects were instructed to describe the colors of the squares in order of appearance after the end of the presentation of the third square. The subjects pushed the left button for blue, the middle button for yellow, and the right button for red.

In each run, a $12 \mathrm{~s}$ baseline period was added before the first task block, and a $15 \mathrm{~s}$ baseline period was added after the third task block. No baseline period was added between the task blocks. During the baseline condition, the subjects were instructed to relax and fixate on the white cross on the screen while their fingers were placed on the smooth portion of the surface beside the gratings. Altogether, 33 volumes (eight volumes times three tasks plus nine volumes for baseline) were collected during each run of the tactile tasks.

\section{Visual task}

The orientation task and its own control condition were performed visually to test for the existence of a multisensory subregion for orientation classification within the IPS. Because the visual task design was different from the tactile design in the timing of responses (see Discussion for an explanation of why this was the case), the visual orientation task was contrasted with its own sensorimotor control condition (color task), which contained the same timing of responses as the visual orientation task. To discourage the subjects from imagining the gratings visually during the tactile tasks, the visual task was performed after the tactile tasks were completed (Fig. 2). The response hand and the order of the tasks were counterbalanced within the subjects. Subjects completed two runs, each of which included six repetitions of each task (Fig. $2 B$ ).

Visual orientation task $\left(V_{O}\right)$. The subjects were instructed to classify the orientation of the linear gratings presented on the screen. A single task block consisted of both instruction $(3 \mathrm{~s})$ and test ( $9 \mathrm{~s}$ ) periods (Fig. $2 C$, top). The instruction cue (viewing angle, $3.6 \times 6.4^{\circ}$ ) was presented in Chinese characters for the first second of the instruction period. During the test period, six gratings were presented (viewing angle, $8.0 \times 8.0^{\circ}$ ) (Fig. $2 \mathrm{~A}$ ). The stimuli had different orientations $\left(-30,0\right.$, and $\left.+30^{\circ}\right)$ and different colors (blue, yellow, and red). The orientation of the gratings changed six times, whereas the color of the gratings changed only once during each test period. Each stimulus was presented for $300 \mathrm{~ms}$, and the interstimulus interval was $1200 \mathrm{~ms}$. Immediately after the presentation of each individual grating, the subjects were asked to press the buttons assigned to each orientation. A single test block was always followed by a $9 \mathrm{~s}$ baseline period (Fig. $2 \mathrm{~B}$ ). During the baseline condition, the subjects were instructed to relax and fixate on the white cross on the screen. The subjects responded using the same buttons as in the tactile orientation task.

Sensorimotor control (color task, $V_{S M}$ ). This condition was designed to control for the sensory input, responses, and task demands of the visual orientation task. The experimental design was the same as in the orientation task, except for the arrangement of stimuli and the task instruction (Fig. 2C, bottom). During the test period, six gratings were presented (Fig. 2A). The color of the gratings changed six times, whereas the orientation of the gratings changed only once during each test period. The subjects were instructed to press the button assigned to each color immediately after the presentation of each individual grating. A $12 \mathrm{~s}$ base-

C Task block

Orientation task $\left(\mathrm{V}_{\mathrm{O}}\right)$
B Task schedule

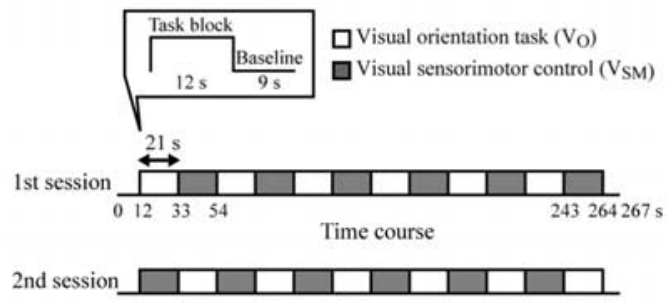

Figure 2. The visual tasks. $\boldsymbol{A}$, Visual stimuli. We used nine different images of gratings, each of which had one of three different Time course ne included six repetitions of each task. Each task block was followed by a $9 \mathrm{~s}$ baseline period. Baseline periods were also added before the final task block in a run. $C$, Task block. The subjects judged the orientation of the gratings during subjects responded by pushing buttons with the fingers of either the left or the right hand. The subjects responded with the same buttons as in the tactile tasks. ISI, Interstimulus interval.

line period was added before the first task block, and a $3 \mathrm{~s}$ baseline period was added after the final task block (five volumes). Altogether, 89 volumes [five baseline volumes + two tasks $\times$ six repetitions $\times$ (four volumes per task block + three volumes per baseline)] were obtained in each run. The subjects pressed the same response buttons as in the tactile motor control task.

\section{Data acquisition and processing}

Vertical force. The vertical pressure of the fingertip on the surface was measured. The bore of the plastic frame was supported by a horizontal acrylic bar, to which two sheets of foil strain gauges were attached (KFP120-C1-65; Kyowa Electronic Instruments, Tokyo, Japan). The force signals were sampled at $100 \mathrm{~Hz}$ and acquired with a personal computer (Thinkpad R51; IBM, Tokyo, Japan) through an amplifier (CDA-700A; Kyowa Electronic Instruments) and an external analog-to-digital converter (MP100A; BioPac Systems, Colorado Springs, CO). The force signals were filtered with a $10 \mathrm{~Hz}$ low-pass filter. The acquired data were further processed with Windows-based software (Acknowledge 3.7.0; BioPac Systems, Goleta, CA).

MRI. Functional MR images were acquired on a 3 tesla head scanner (Allegra; Siemens, Erlangen, Germany) with echoplanar imaging (EPI) capability. Standard sequence parameters were used for obtaining the functional images as follows: gradient-echo EPI; repetition time, 3000 $\mathrm{ms}$; echo time, $30 \mathrm{~ms}$; flip angle, $85^{\circ}$; 44 axial slices of $3 \mathrm{~mm}$ thickness with 


\begin{tabular}{|c|c|c|c|c|c|c|}
\hline \multirow[b]{3}{*}{ Name of contrasts } & \multicolumn{6}{|l|}{ Conditions } \\
\hline & \multicolumn{3}{|l|}{ Left hand } & \multicolumn{3}{|l|}{ Right hand } \\
\hline & Orientation & Sensorimotor control & Motor control & Orientation & Sensorimotor control & Motor control \\
\hline$T_{0}-T_{M}$ & 1 & 0 & -1 & 1 & 0 & -1 \\
\hline $\mathrm{T}_{\mathrm{SM}}-T_{M}$ & 0 & 1 & -1 & 0 & 1 & -1 \\
\hline$T_{O}-T_{S M}$ & 1 & -1 & 0 & 1 & -1 & 0 \\
\hline$T_{S M}-T_{0}$ & -1 & 1 & 0 & -1 & 1 & 0 \\
\hline Left hand $\left(T_{0}-T_{S M}\right)$ & 1 & -1 & 0 & 0 & 0 & 0 \\
\hline Right hand $\left(T_{O}-T_{S M}\right)$ & 0 & 0 & 0 & 1 & -1 & 0 \\
\hline
\end{tabular}

no interslice gap; field of view, $192 \times 192 \mathrm{~mm}$; and in-plane resolution, $3.0 \times 3.0 \mathrm{~mm}$. After the acquisition of functional images, T1-weighted high-resolution anatomical images were obtained (voxel size, $0.9 \times$ $0.9 \times 1 \mathrm{~mm}$ ). Image processing and statistical analyses were performed using the statistical parametric mapping (SPM) package (SPM99; http:// www.fil.ion.ucl.ac.uk/spm; Wellcome Department of Cognitive Neurology, London, UK) implemented in MATLAB (MathWorks, Sherborn, MA) (Friston et al., 1995a,b). The first five volumes of each fMRI run were discarded because of unsteady magnetization. Realigned images were normalized to a standard EPI template as defined by the Montreal Neurological Institute; this closely approximates to the space described in the Talairach and Tournoux (1988) atlas. The normalized EPI images were filtered using a Gaussian kernel of $8 \mathrm{~mm}$ full-width at halfmaximum in the $x, y$, and $z$ axes. The T1-weighted high-resolution anatomical images were normalized by the same procedure.

\section{Statistical analysis}

Behavioral data were collected and statistically evaluated with SPSS software (version 10.0J; SPSS Japan, Tokyo, Japan). Statistical analyses of fMRI data were conducted at two levels. First, individual task-related activation was evaluated. Second, to make inferences at a population level, individual data were summarized and incorporated into a randomeffect model (Holmes and Friston, 1998; Friston et al., 1999).

Individual analysis. We fitted a general linear model to the functional MRI data from each subject (Friston et al., 1994; Worsley and Friston, 1995). The time series for each voxel was high-pass filtered to $0.021 \mathrm{~Hz}$ and low-pass filtered by a canonical hemodynamic-response function. In the tactile tasks, the neural activity during the instruction periods, response periods, and three test periods were each modeled with boxcar functions convolved with a canonical hemodynamic-response function. Twenty-four runs (12 right-handed and 12 left-handed) were included in the group-design matrix for each subject. Each run included five regressors of a boxcar function: a regressor for the instruction period of all of the task conditions, a regressor for the response period, and three regressors for each test period of the task conditions. In contrast, the two runs of the visual task were included in the separate group-design matrix of each subject. The neural activities for the instruction and test periods were modeled, each with boxcar functions convolved with a canonical hemodynamic-response function. Each run included three regressors: a regressor for the instruction of all the conditions and two regressors for each test period of the conditions.

To test hypotheses about regionally specific condition effects, the estimates for each condition were compared by means of linear contrasts. The resulting set of voxel values for each comparison constituted an SPM of the $t$ statistic [SPM $\{t\}]$. The SPM $\{t\}$ was transformed to normal distribution units $[\operatorname{SPM}\{z\}]$. In the tactile tasks, we first evaluated the contrast between the orientation and motor control conditions $T_{O}-T_{M}$, and the contrast between the sensorimotor control and motor control conditions $T_{S M}-T_{M}$ within the whole brain, to confirm that the somatosensory cortex was activated during tactile stimulation (Table 1). We then performed the contrast between the orientation and sensorimotor control conditions $T o-T_{S M}$ in the whole brain to evaluate the neural substrates for the spatial processing of orientation classification. The opposite contrast $T_{S M}-T o$ was also performed in the whole brain. The contrast To$T_{S M}$ was also performed to compare the left and right hands, to further examine any differences in brain activity attributable to the hand used to perform the task.

To examine the multisensory activity in the IPS area, the contrast between the visual orientation versus the control condition $\left(V_{O}-V_{S M}\right)$ was evaluated within the areas highlighted by contrasting the tactile orientation task and the sensorimotor control condition $\left(T o-T_{S M}\right)$. The opposite contrast $V_{S M}-V o$ was also performed in the whole brain. The threshold for $\operatorname{SPM}\{z\}$ was set at $Z>2.33$.

Group analysis with random-effect model. The weighted sum of the parameter estimates in an individual analysis constituted contrast images, which were used for the group analysis (Holmes and Friston, 1998; Friston et al., 1999). At the group level, we performed the same linear contrasts as in the individual analyses (Table 1). The contrast images obtained from the individual analyses represent the normalized taskrelated increment of the MR signal of each subject. For each contrast, a one-sample $t$ test was performed for every voxel in the brain to obtain population inferences. The resulting set of voxel values for each contrast constituted the $\operatorname{SPM}\{t\}$. The SPM $\{t\}$ was transformed to normal distribution units $[\operatorname{SPM}\{z\}]$.

To evaluate the hemisphere effects on tactile spatial processing, contrast images of $T_{O}-T_{S M}$ (Table 1) were flipped in the horizontal (rightleft) direction. Asymmetric involvement of the neural substrates for tactile spatial processing, regardless of the hand used, was shown by the comparison between the unflipped and flipped images in a pairwise manner (Harada et al., 2004). The comparison was performed within the regions that showed activation in the $T_{O}-T_{S M}$ contrast.

To examine multisensory activity in the IPS area, the visual orientation versus control conditions contrast $V_{O}-V_{S M}$ was evaluated within the areas that showed activation by the contrast of $T_{O}-T_{S M}$. The threshold for $\operatorname{SPM}\{z\}$ was set at $Z>2.33$. The statistical threshold for the spatial extent test on the clusters was set at $p<0.05$ and corrected for multiple comparisons within the search volume (Friston et al., 1996). The thresholds of the spatial extent test were as follows: $5656 \mathrm{~mm}^{3}$ for $T_{O}-T_{M}$; $4320 \mathrm{~mm}^{3}$ for $T_{S M}-T_{M} ; 4240 \mathrm{~mm}^{3}$ for $T_{O}-T_{S M}$ and $T_{S M}-T_{O}$; $4000 \mathrm{~mm}^{3}$ for the left hand $\left(T_{O}-T_{S M}\right) ; 3800 \mathrm{~mm}^{3}$ for the right hand $\left(T_{O}-T_{S M}\right) ; 744 \mathrm{~mm}^{3}$ for the hemispheric laterality test; and 1584 $\mathrm{mm}^{3}$ for $V_{O}-V_{S M}$.

\section{Results}

\section{Task performance}

\section{Tactile tasks}

The subjects were able to classify the gratings equally well, regardless of the task or which hand was used (Fig. 3A). A two-way repeated-measures ANOVA [(three task conditions: tactile orientation, sensorimotor control, and motor control) $\times$ (two hands: left and right)] on the accuracy scores (percentage correct) showed a significant effect of condition $\left(F_{(2,30)}=20.5 ; p<\right.$ $0.001)$. Bonferroni's $t$ tests for multiple comparisons showed significant differences only between the motor control and the other conditions $(p<0.001)$. The response times were also similar between the orientation $\left(T_{O}\right)$ and sensorimotor control tasks $\left(T_{S M}\right)$ (Fig. $3 B$ ). A three-way repeated measures ANOVA [(three task conditions: tactile orientation, sensorimotor control and 
A Accuracy

B Response time
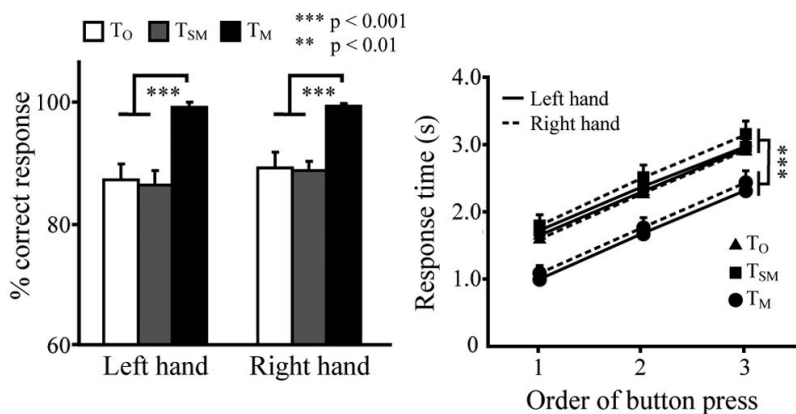

C Vertical pressure

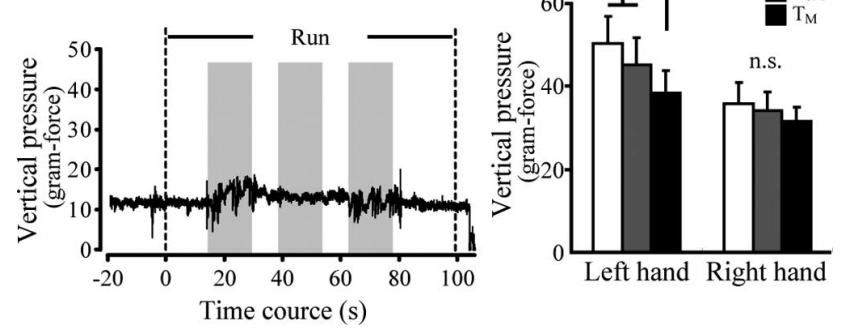

Figure 3. Behavioral results. A, Accuracy of performance on the tactile tasks. There was little difference between the orientation tasks and the sensorimotor control conditions; however, subjects responded more accurately during the motor control condition than during the other conditions ( $p<0.001)$. $\boldsymbol{B}$, Response times of the three button presses. The response times during the motor control condition were significantly shorter than during the other conditions $(p<0.001)$, whereas there was no significant difference between the orientation and sensorimotor control conditions. C, Vertical pressure of the middle finger. Left, An example of recordings of vertical pressure during a single run is shown. The shaded square indicates the test periods. Right, There was a significant difference in the vertical pressure applied with the left finger between the motor control and other conditions ( $p<0.01$ ). However, only negligible differences were observed between the orientation task and the sensorimotor control conditions, regardless of the hand used. These data are presented as the mean \pm SEM of 16 subjects. n.s., Not significant.

motor control $) \times($ two hands: left and right $) \times$ (order of response: first, second, and third button press)] of the response times showed significant effects of condition $\left(F_{(2,30)}=22.0 ; p<\right.$ $0.001)$ and $\operatorname{order}\left(F_{(2,30)}=249 ; p<0.001\right)$. Bonferroni's $t$ tests for multiple comparisons showed significant differences between the motor control and the other conditions $(p<0.001)$.

The vertical pressure applied by the right finger was nearly constant between conditions, whereas the pressure exerted by the left finger varied by condition (Fig. 3C). The same repeatedmeasures ANOVA performed on the vertical pressure showed a significant condition $\times$ hand interaction $\left(F_{(2,30)}=4.92 ; p=\right.$ $0.014)$. Multiple comparisons showed significant differences between the motor control and the other conditions $(p<0.01)$ for the left hand. However, there were no significant differences between the tactile orientation task and sensorimotor control condition for either the right hand $(p>0.9)$ or the left hand $(p=0.064)$.

\section{Visual tasks}

The subjects classified the gratings equally well, regardless of the task. Performance accuracy was matched between the conditions (mean $\pm \mathrm{SEM}=98.0 \pm 0.5 \%$ for the orientation condition and $97.1 \pm 0.8 \%$ for the control condition). A Student's $t$ test showed no significant difference between the accuracy of responses $(p>$ $0.3)$. Response times for the orientation task were marginally faster than for the sensorimotor control condition $(527.4 \pm 16.8$ $\mathrm{ms}$ for the orientation task and $557.9 \pm 24.3 \mathrm{~ms}$ for the control condition). A Student's $t$ test showed a significant difference between these response times $\left(t_{(15)}=2.39 ; p=0.030\right)$; however, the difference was only $5.7 \%$, which was negligible within the context of this study.

\section{fMRI results}

Comparisons between the tactile task and motor control conditions $\left(\mathrm{T}_{O}-T_{M}\right.$ and $\left.T_{S M}-T_{M}\right)$

Table 2 shows the coordinates of the foci in the significantly activated areas. The contrast of the tactile orientation versus motor control condition $\left(T_{O}-T_{M}\right)$ significantly activated the postcentral gyrus (presumably the primary somatosensory cortex), parietal operculum (PO), posterior insula, medial frontal cortex, lateral frontal cortex, posterior parietal cortex, precuneus, basal ganglia, and cerebellum bilaterally. This contrast also activated the right lateral prefrontal cortex, orbitofrontal cortex, and anterior insula (Table 2, Fig. 4AI). The contrast of the tactile sensorimotor control versus motor control condition $\left(T_{S M}-T_{M}\right)$ activated the postcentral gyrus, $\mathrm{PO}$, anterior and posterior insula, lateral prefrontal cortex, medial and lateral frontal cortex, posterior parietal cortex, precuneus, basal ganglia, and cerebellum bilaterally, and the right orbitofrontal cortex (Table 2, Fig. 4AII).

Comparisons between the tactile orientation and sensorimotor control conditions $\left(\mathrm{T}_{O}-T_{S M}\right.$ and $\left.T_{S M}-T_{O}\right)$

When the tactile orientation task was contrasted with its sensorimotor control condition $\left(T_{O}-T_{S M}\right)$, the areas around the IPS were activated bilaterally (Table 2, Fig. 4AIII). The cluster in each hemisphere extended from the postcentral sulcus anteriorly to the posterior part of the IPS posteriorly. The right cluster was conspicuously larger than the left cluster $\left(17,912 \mathrm{~mm}^{3}\right.$ for the right hemisphere and $4712 \mathrm{~mm}^{3}$ for the left hemisphere). When the $\left(T_{O}-T_{S M}\right)$ contrasts were performed for each hand, areas around the right IPS were activated regardless of the hand used. The overlapping area between the two hands was located in the right post-central sulcus (CS) and IPS (volume, $4720 \mathrm{~mm}^{3}$; coordinates for center of mass: $x=38, y=-47$, and $z=56$ ). When the sensorimotor control condition was contrasted with the orientation task, the contrast activated the bilateral lateral prefrontal cortices, the right anterior insula, orbitofrontal cortex, lingual gyrus, basal ganglia, amygdala, and the left lingual/fusiform gyrus, inferior/middle occipital gyrus, and cerebellum (Table 2, Fig. $4 A I V$ ). The left PO (coordinates: $x=54, y=-14, z=14 ; Z$ value $=3.20$ ) and right posterior insula (coordinates: $x=38, y=$ $-12, z=2 ; Z$ value $=3.06$ ) were also activated, but the cluster size of these areas did not reach the statistical threshold.

\section{Hemispheric effect}

Right-lateralized activity was found in a cluster extending from the post-CS to the anterior part of the IPS (volume, $1736 \mathrm{~mm}^{3}$ ). The peak of the cluster was $Z=3.79$ at the coordinates $x=48$, $y=-42$, and $z=52$ (Fig. 5).

\section{Multisensory activation in the IPS for the classification of} grating orientation

When the visual orientation task was contrasted with the sensorimotor-control condition $\left(V_{O}-V_{S M}\right)$, the left middle occipital gyrus (MOG) was significantly activated (Table 3 ). This contrast also showed activation in a part of the right IPS within the cluster activated by the tactile orientation task $\left(T_{O}-T_{S M}\right.$ ) (Fig. 6). The area of overlap extended from the anterior to the middle part of the IPS. The volume of this area was $1704 \mathrm{~mm}^{3}$ with the center of mass at coordinates $x=35$, $y=-51$, and $z=58$. Figure 7 shows two representative 
Table 2. Task-related activity of the tactile tasks $(n=16)$

\begin{tabular}{|c|c|c|c|c|c|c|c|c|c|}
\hline \multirow[b]{2}{*}{ Anatomical region } & \multirow[b]{2}{*}{ Hem } & \multicolumn{3}{|c|}{$T_{0}-T_{M}$} & \multirow[b]{2}{*}{$Z$ value } & \multicolumn{3}{|c|}{$T_{S M}-T_{M}$} & \multirow[b]{2}{*}{$Z$ value } \\
\hline & & $x$ & $y$ & $z$ & & $x$ & $y$ & $z$ & \\
\hline \multirow[t]{2}{*}{ Postcentral gyrus } & $\mathrm{L}$ & -54 & -26 & 50 & 5.15 & -54 & -26 & 50 & 4.95 \\
\hline & $\mathrm{R}$ & 52 & -34 & 58 & 4.97 & 58 & -28 & 50 & 4.53 \\
\hline \multirow[t]{2}{*}{ Parietal operculum } & $\mathrm{L}$ & -60 & -24 & 26 & 4.74 & -58 & -22 & 22 & 5.16 \\
\hline & $\mathrm{R}$ & 56 & -20 & 20 & 4.95 & 54 & -22 & 18 & 4.82 \\
\hline \multirow[t]{2}{*}{ Posterior insula } & $L$ & -42 & -8 & 8 & 4.65 & -40 & -4 & -4 & 5.08 \\
\hline & $\mathrm{R}$ & 38 & 2 & 12 & 3.34 & 40 & -10 & 6 & 4.10 \\
\hline \multirow[t]{2}{*}{ Anterior insula } & $\mathrm{L}$ & & & & & -38 & 16 & -4 & 5.35 \\
\hline & $\mathrm{R}$ & 32 & 24 & 10 & 5.80 & 34 & 22 & -6 & 6.00 \\
\hline \multirow[t]{2}{*}{ Lateral prefrontal cortex } & $\mathrm{L}$ & & & & & -40 & 28 & 24 & 4.87 \\
\hline & $\mathrm{R}$ & 52 & 30 & 32 & 5.33 & 52 & 32 & 28 & 6.60 \\
\hline \multirow[t]{2}{*}{ Medial frontal cortex } & $\mathrm{L}$ & -4 & 14 & 52 & 3.92 & -4 & 16 & 54 & 4.70 \\
\hline & $\mathrm{R}$ & 4 & 18 & 48 & 4.45 & 4 & 20 & 52 & 5.38 \\
\hline \multirow[t]{2}{*}{ Dorsolateral frontal cortex } & $L$ & -20 & 0 & 54 & 4.45 & -24 & 2 & 54 & 5.37 \\
\hline & $\mathrm{R}$ & 30 & 2 & 62 & 4.67 & 34 & 4 & 58 & 4.95 \\
\hline \multirow[t]{2}{*}{ Ventrolateral frontal cortex } & $\mathrm{L}$ & -54 & 6 & 22 & 4.95 & -46 & 4 & 30 & 5.44 \\
\hline & $\mathrm{R}$ & 58 & 8 & 22 & 5.30 & 50 & 10 & 24 & 4.49 \\
\hline Orbitofrontal cortex & $\mathrm{R}$ & 32 & 50 & -4 & 3.09 & 32 & 50 & -4 & 3.96 \\
\hline \multirow[t]{2}{*}{ Posterior parietal cortex } & $\mathrm{L}$ & -38 & -48 & 56 & 5.21 & -36 & -48 & 56 & 5.12 \\
\hline & $\mathrm{R}$ & 36 & -52 & 56 & 4.67 & 34 & -44 & 70 & 4.46 \\
\hline \multirow[t]{2}{*}{ Precuneus } & $\mathrm{L}$ & -10 & -74 & 50 & 3.74 & -14 & -76 & 44 & 3.66 \\
\hline & $\mathrm{R}$ & 12 & -70 & 50 & 4.23 & 12 & -70 & 50 & 4.20 \\
\hline \multirow[t]{2}{*}{ Basal ganglia } & $\mathrm{L}$ & -16 & -6 & 0 & 3.51 & -10 & -2 & 0 & 4.43 \\
\hline & $\mathrm{R}$ & 14 & 6 & 2 & 3.58 & 12 & 10 & 2 & 4.31 \\
\hline \multirow[t]{3}{*}{ Cerebellum } & $L$ & -18 & -76 & -26 & 4.78 & -22 & -50 & -32 & 4.69 \\
\hline & $\mathrm{R}$ & 36 & -42 & -40 & 5.60 & 30 & -58 & -30 & 4.20 \\
\hline & & \multicolumn{3}{|c|}{$T_{0}-T_{S M}$} & & $T_{S M}$ & & & \\
\hline Postcentral gyrus & $\mathrm{R}$ & 50 & -36 & 60 & 3.49 & & & & \\
\hline \multirow[t]{2}{*}{ Intraparietal sulcus } & $L$ & -24 & -56 & 58 & 3.93 & & & & \\
\hline & $\mathrm{R}$ & 34 & -58 & 58 & 4.15 & & & & \\
\hline \multirow[t]{2}{*}{ Lateral prefrontal cortex } & $\mathrm{L}$ & & & & & -28 & 42 & 36 & 3.91 \\
\hline & $\mathrm{R}$ & & & & & 48 & 38 & 18 & 4.20 \\
\hline Anterior insula & $\mathrm{R}$ & & & & & 38 & 12 & -6 & 3.04 \\
\hline Orbitofrontal cortex & $\mathrm{R}$ & & & & & 16 & 34 & -20 & 2.87 \\
\hline Basal ganglia & $\mathrm{R}$ & & & & & 20 & 8 & -8 & 3.78 \\
\hline Amygdala & $\mathrm{R}$ & & & & & 22 & -2 & -14 & 3.24 \\
\hline Lingual/fusiform gyrus & $L$ & & & & & -26 & -72 & -6 & 3.77 \\
\hline Lingual gyrus & $\mathrm{R}$ & & & & & 10 & -76 & 2 & 4.29 \\
\hline Inferior/middle occipital gyrus & $\mathrm{L}$ & & & & & -30 & -80 & -8 & 3.63 \\
\hline Cerebellum & $\mathrm{L}$ & & & & & -32 & -72 & -18 & 3.53 \\
\hline
\end{tabular}

Hem, Hemisphere; L, left; $R$, right; $x, y$, and $z$ are stereotaxic coordinates $(\mathrm{mm}) ; p<0.05$ at the cluster level corrected for multiple comparisons.

examples from the analyses of the individual data. No significant activation was observed when the sensorimotor control condition was contrasted with the orientation task $\left(V_{S M}-V_{O}\right)$.

\section{Discussion}

The right post-CS and the IPS regions were activated when the tactile orientation task was contrasted with the sensorimotor control condition (roughness task), regardless of the hand used. In contrast, when contrasted with its control condition (color task), the visual orientation task activated the left MOG. As hypothesized, a part of the right IPS was activated by both the tactile and visual orientation tasks and hence might represent a multisensory processing area.

\section{Task design and behavioral performance}

In the tactile pilot experiment, perceived roughness magnitude varied with grating orientation. Gratings feel rougher when they move perpendicular to, as opposed to along, the gratings. The roughness percept is likely enhanced by the skin consistently catching on the leading edges as the gratings are moved directly across the finger. The difference between the two other orientations may also be explained by mechanical effects attributable to differential skin catching.

The main task-design difference between the sensory modalities was in the timing of responses. The subjects responded after the presentation of each grating in the visual task, whereas in the tactile task they responded to all three gratings only after the presentation of the third surface. This variation derives from the nature of the tactile sensorimotor control task: the classification of surface roughness. Although subjects can rely on some form of spatial reference system to classify grating orientation, they must rely on the perceived roughness of the other surfaces to classify the gratings in terms of perceived roughness. Subjects in a pilot study showed poor performance in roughness classification when 
A
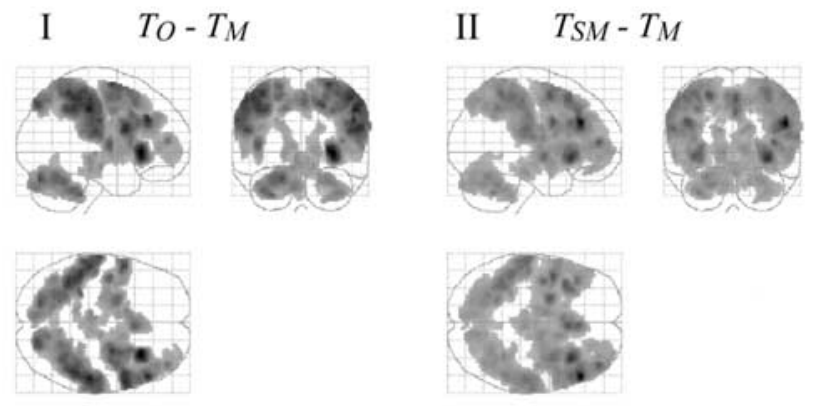

III $\quad T_{O}-T_{S M}$
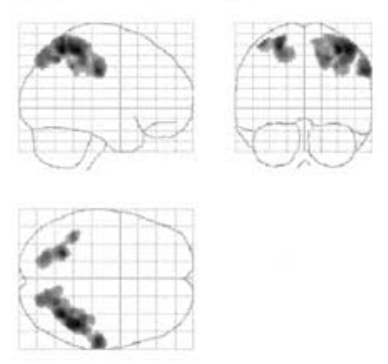

IV $T_{S M}-T_{O}$

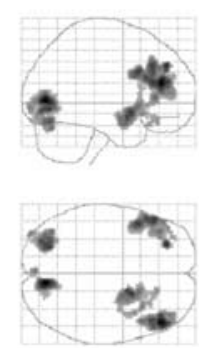

B

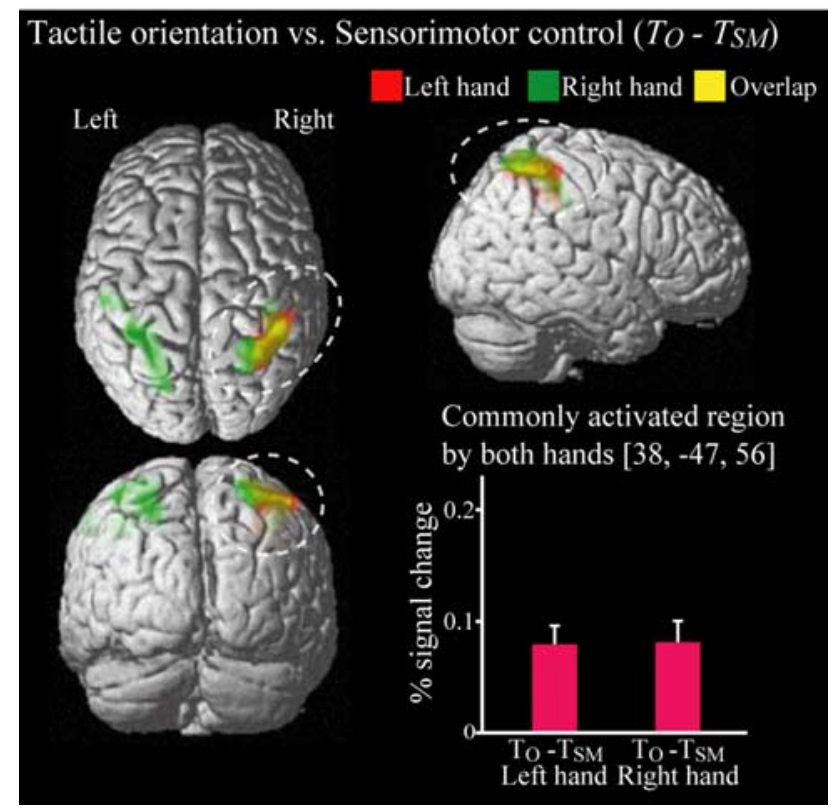

Figure 4. Activation patterns in the tactile tasks. $\boldsymbol{A}$, Statistical parametric map of the average neural activity within the group during the orientation task compared with the motor control $\operatorname{task}\left(T_{O}-T_{M}\right)$, the sensorimotor control compared with the motor control task $\left(T_{S M}-T_{M}\right)$, the orientation task compared with the sensorimotor control $\left(T_{O}-T_{S M}\right)$, and the sensorimotor control compared with the orientation task $\left(T_{S M}-T_{0}\right)$. The three-dimensional information was collapsed into two-dimensional sagittal, coronal, and transverse images (i.e., maximumintensity projections viewed from the right, back, and top of the brain). $\boldsymbol{B}$, The activation patterns during the orientation task when performed with the left (red) and right (green) hands were superimposed on surface-rendered high-resolution MRIs unrelated to the subjects of the present study, viewed from the top, right, and back of the brain. The right post-CS and IPS were activated by the contrast of $\left(T_{O}-T_{S M}\right)$, regardless of the hand used (yellow within white dashed circle). Bar graphs indicate the difference in activity (percentage signal change) between the orientation task $\left(T_{0}\right)$ and sensorimotor control conditions $\left(T_{S M}\right)$ in the region of the right IPS, using a volume of interest with a sphere of $8 \mathrm{~mm}$ diameter The center of the sphere was the center of mass for the common activation during task performance with both hands. These data are presented as the mean \pm SEM of 16 subjects.

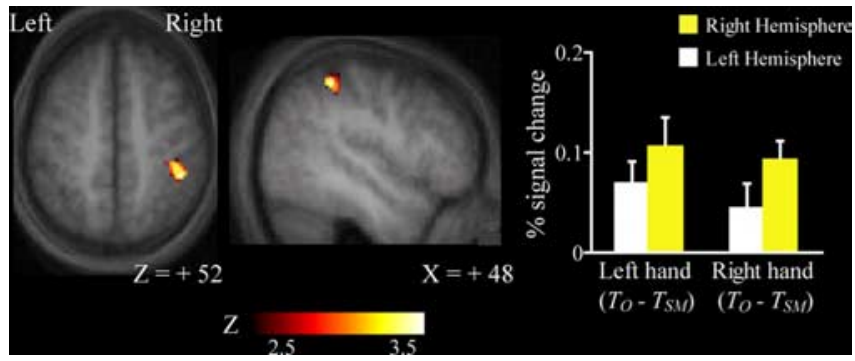

Figure 5. Asymmetrical neural representation of the tactile orientation classification by either hand. The contrast images of $T_{O}-T_{S M}$ were compared with those flipped in the horizontal (right-left) direction in a pairwise manner (Table 1). The test was performed within the areas that revealed activation by the contrast of $T_{O}-T_{S M}$. The statistical parametric map was superimposed on transverse and sagittal images, which represent the mean of the T1-weighted high-resolution MRIs for the subjects. Bar graphs indicate the difference in signal change between conditions in the right IPS and left IPS using a volume of interest with a sphere of $8 \mathrm{~mm}$ diameter. The centers of the spheres were the peak coordinates of activation in the right hemisphere and the flipped coordinates in the left hemisphere, respectively. These data are presented as the mean \pm SEM of 16 subjects.

there were no reference surfaces. In contrast, in the visual task, if we had used the same timing of responses as in the tactile task, there would have been substantial demands on memory: the subjects would have had to remember more responses because of the shorter duration of the presentation of visual stimuli.

The present experiment was designed to examine the existence of a multisensory area activated by both visual and tactile orientation tasks, as opposed to directly comparing the two modalities. Therefore, the orientation task for each sensory modality was contrasted with its own control condition, in which subjects were given the same sensory input, responded at the same time, and used the same fingers. In tactile tasks, furthermore, we excluded any activity during the response period from the taskrelated activity (test period) by using separate regressors. Collectively, the difference in the timing of responses between the sensory modalities should not explain the multisensory activation in the IPS.

It is known that the parietal-premotor cortical network is related to top-down attentional modulation (Corbetta, 1998; Hopfinger et al., 2000). In the present study, multiple brain areas were activated, when the tactile tasks were compared with the motor control task. Because the motor control task was easier than the other conditions, the activation might reflect difference in attentional demands as well as in the sensory and cognitive processing between the task conditions. In contrast, multisensory activation for the orientation judgment was only found in the IPS. In the tactile tasks, sequential finger responses were easy to perform, because the motor control task showed virtually perfect task accuracy. Because task accuracy was similar at $<90 \%$ between the other tactile tasks, we can assume that attentional demands were comparable between them. Furthermore, the visual orientation task showed a significantly lower response time than its sensorimotor control task. This result indicates that more attentional demand, if any, might have been required in the control than in the orientation task. Collectively, it is unlikely that multisensory activation is caused by higher attentional demands in the orientation task than in its own sensorimotor control.

In the orientation task, subjects needed to rely on a spatial reference system to classify the orientation of the gratings. In contrast, it was necessary to use other cues in the control condi- 
Table 3. Task-related activity of the visual orientation task $(n=16)$

\begin{tabular}{|c|c|c|c|c|c|c|c|c|c|}
\hline \multirow[b]{2}{*}{ Anatomical region } & \multirow[b]{2}{*}{ Hem } & \multicolumn{3}{|c|}{$V_{0}-V_{S M}$} & \multirow[b]{2}{*}{$Z$ value } & \multicolumn{3}{|c|}{$V_{S M}-V_{O}$} & \multirow[b]{2}{*}{ Zvalue } \\
\hline & & $x$ & $y$ & $z$ & & $x$ & $y$ & $z$ & \\
\hline Middle occipital gyrus & $\mathrm{L}$ & -40 & -84 & 10 & 4.15 & & & & \\
\hline Intraparietal sulcus & $\mathrm{R}$ & 30 & -54 & 56 & 3.56 & & & & \\
\hline
\end{tabular}

The contrast of $V_{S M}-V_{O}$ did not yield any significant cluster of activation. Hem, Hemisphere; $L$, left; $R$, right.

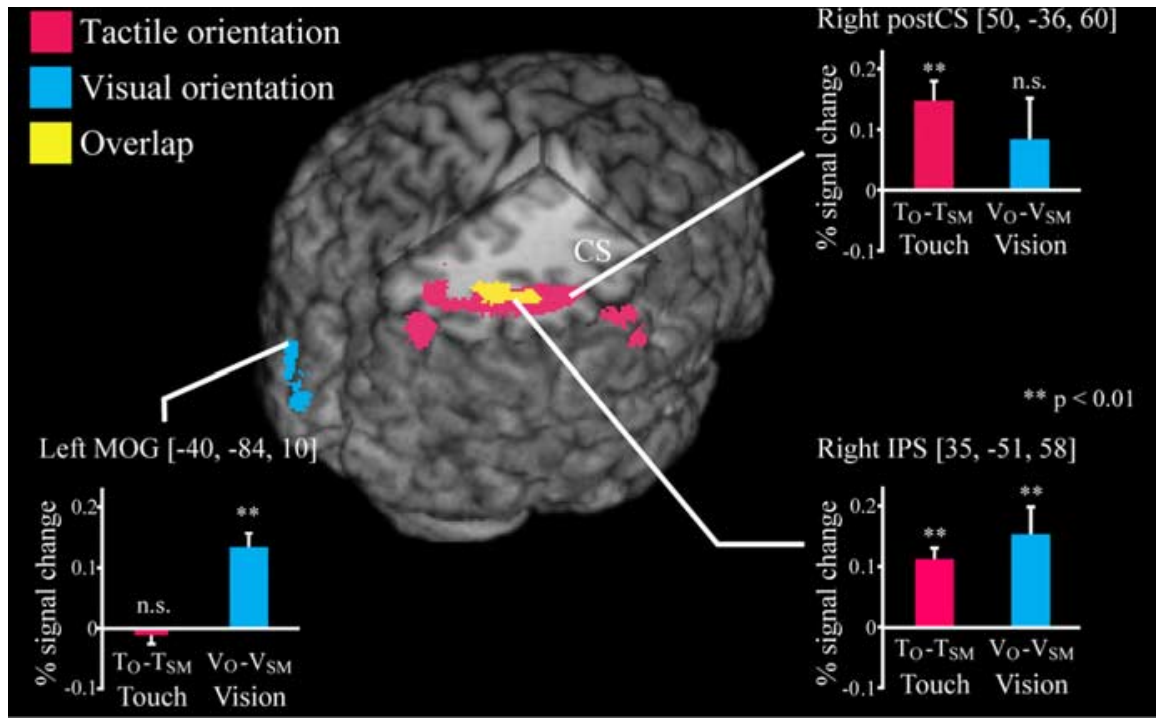

Figure 6. Multisensory activation in the IPS. The statistical parametric map of the average neural activity within the group during the visual orientation task compared with the activity during the sensorimotor control condition $\left(V_{O}-V_{S M}\right)$ were depicted within the regions activated by $T_{O}-T_{S M}$ in the tactile conditions. The SPM was superimposed on a surface-rendered high-resolution MRI unrelated to the subjects of the present study. Bar graphs indicate the difference in activity (percentage signal change) between the orientation task and sensorimotor control condition using a volume of interest with a sphere of $8 \mathrm{~mm}$ diameter. The centers of the spheres were the peak coordinates of activation. ${ }^{* *}$ Statistically significant difference ( $p<0.01$, one-sample $t$ test). These data are presented as the mean \pm SEM of 16 subjects. n.S., Not significant.

tions (i.e., roughness magnitude for tactile and color for visual modalities). Hence, contrasting the orientation tasks with their sensorimotor control conditions should highlight the spatial processing required during orientation classification.

\section{Sensory-specific activation}

The right post-CS and anterior IPS were activated by the tactile orientation judgment, regardless of the hand used. The asymmetric activation in these areas corresponded with the results of Harada et al. (2004), which showed right-lateralized activation in the IPS during the tactile discrimination of two-dot spatial patterns. These areas might play an important role in the extraction of spatial information such as grating orientation from the anterior part of the postcentral gyrus.

In contrast, the visual orientation task specifically activated the left MOG. This result confirms the findings of Faillenot et al. (2001), which showed left MOG activation during the visual discrimination of grating orientation. The MOG was also activated by other visuospatial tasks, including the judgment of line orientation (Kesler et al., 2004) and mental rotation tasks (Podzebenko et al., 2002). The MOG might work in concert with other cortical areas, such as the IPS, for the visuospatial processing of orientation.

\section{Multisensory activation}

The main finding of the current study is that the multisensory orientation judgment of gratings activated a subregion of the IPS.
It has been known that the IPS is important for visual orientation judgment (Eacott and Gaffan, 1991). For instance, neurons in the anterior IPS of nonhuman primates are visually selective to the orientation of objects (Murata et al., 2000), whereas neurons in the posterior IPS are tuned for orientation in the frontoparallel plane of elongated objects (Sakata and Taira, 1994). In humans, part of the IPS was also activated during the visual discrimination of grating orientation (Vandenberghe et al., 1996; Faillenot et al., 2001).

In contrast, it has been unclear how this region is involved in tactile orientation judgment. Previous studies indicated that the extrastriate area might be crucial for tactile orientation judgment, because this area was activated by orientation judgment (Sathian et al., 1997; Zangaladze et al., 1999). However, recent studies also reported activation of the IPS during the tactile orientation judgment of gratings (Van Boven et al., 2005; Zhang et al., 2005). The findings from both visual and tactile studies indicate that the IPS might be crucial for the multisensory judgment of orientation. However, there is little evidence regarding the existence of a multisensory subregion within the human IPS, which is involved in grating orientation judgment.

The current study extends the findings of previous studies by showing that a subregion of the right IPS is crucial for multisensory orientation judgment. Neural populations in this region might constitute a multisensory orientation-related network. This idea is supported by the results of previous fMRI studies (Grefkes et al., 2002; Saito et al., 2003; Zhang et al., 2004). For instance, Bremmer et al. (2001) showed that a ventral part of the IPS was activated by polysensory motion stimuli around the subject's head. The authors proposed that the IPS subregion encodes sensory information from different sensory modalities in a bodycentered frame of reference.

It is possible that the IPS subregion might integrate the orientation representations from different sensory modalities into a supramodal representation within a single spatial reference system. The sensory-specific areas may extract spatial information of grating orientation from early sensory areas, whereas the IPS subregion may transform such spatial information in terms of a spatial reference system. In the present experiment, each sensory modality could encode grating orientation using several different systems of spatial reference, yet the subjects eventually responded with the same fingers. In other words, the subjects perceived the grating orientations from both sensory modalities by making an orientation judg- 


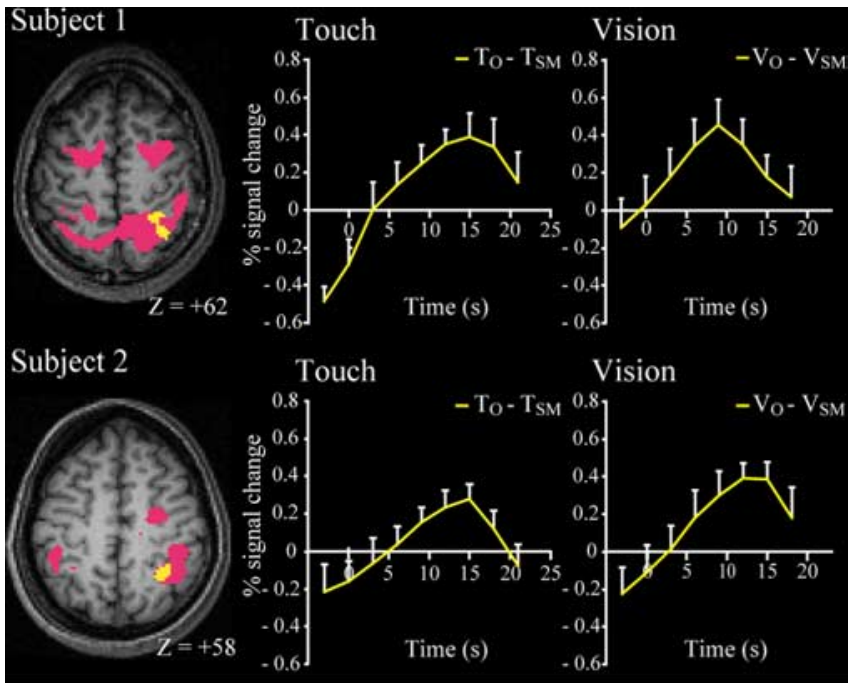

Figure 7. Individual analysis of multisensory activation in the IPS. The brain regions activated by the contrasts of $V_{O}-V_{S M}$ in the visual conditions were depicted (yellow area) within the regions activated by $T_{O}-T_{S M}$ during the tactile conditions (red area). The statistical parametric map was superimposed on the transverse plane of the T1-weighted high-resolution MR for each subject (left column, for each subject). The percentage signal change is plotted as the mean \pm SEM of 12 repetitions for each subject (right columns, for each subject). The yellow line indicates the difference in percentage signal change between the orientation task and sensorimotor control conditions. Each test period started from $0 \mathrm{~s}$

ment in the same type of spatial reference frame. It could be concluded from this that humans might be able to experience the orientation of gratings as both a single and an integrated representation. This hypothesis is supported by the notion that the posterior parietal cortex combines information from different sensory modalities to form a unified representation of space (Andersen et al., 1997). In spatial hemineglect, subjective orientation judgment is disrupted multimodally (Kerkhoff, 2001). Our results are in accordance with this finding, implying that the right IPS might be crucial for orientation judgment.

\section{Visual mediation heuristic}

In contrast, we observed no strong activation of the extrastriate cortex during orientation judgments. According to the hypothesis of Sathian et al. (1997), the differences in results compared with previous studies might derive from the degree to which visual mediation was applied. The subjects in our study might have been able to judge grating orientation without visualizing the gratings as vividly as the subjects in previous studies. Congenitally blind subjects, who have no visual experience, can perform several haptic tasks that require spatial processing, including mental rotation tasks (Marmor and Zaback, 1976; Carpenter and Eisenberg, 1978), estimating the spatial density of textures (Merabet et al., 2004), and recognizing objects such as a mask, shoes, and bottles (Pietrini et al., 2004). These results suggest that the judgment of grating orientations might not always require visual mediation heuristics. In conclusion, a subregion in the middle IPS might play an important role in the multisensory judgment of grating orientation.

\section{References}

Andersen RA, Snyder LH, Bradley DC, Xing J (1997) Multisensory representation of space in the posterior parietal cortex and its use in planning movements. Annu Rev Neurosci 20:303-330.

Bodegard A, Geyer S, Grefkes C, Zilles K, Roland PE (2001) Hierarchical processing of tactile shape in the human brain. Neuron 31:317-328.

Bremmer F, Schlack A, Shah NJ, Zafiris O, Kubischik M, Hoffmann K, Zilles K, Fink GR (2001) Polymodal motion processing in posterior parietal and premotor cortex: a human fMRI study strongly implies equivalencies between humans and monkeys. Neuron 29:287-296.

Carpenter PA, Eisenberg P (1978) Mental rotation and the frame of reference in blind and sighted individuals. Percept Psychophys 23:117-124.

Corbetta M (1998) Frontoparietal cortical networks for directing attention and the eye to visual locations: identical, independent, or overlapping neural systems? Proc Natl Acad Sci USA 95:831-838.

Eacott MJ, Gaffan D (1991) The role of monkey inferior parietal cortex in visual discrimination of identity and orientation of shapes. Behav Brain Res 46:95-98.

Faillenot I, Sunaert S, Van Hecke P, Orban GA (2001) Orientation discrimination of objects and gratings compared: an fMRI study. Eur J Neurosci 13:585-596.

Friston KJ, Jezzard P, Turner R (1994) Analysis of functional MRI timeseries. Hum Brain Mapp 1:153-171.

Friston KJ, Ashburner J, Frith CD, Heather JD, Frackowiak RSJ (1995a) Spatial registration and normalization of images. Hum Brain Mapp 2:165-188.

Friston KJ, Holmes AP, Worsley KJ, Poline J-B, Frith CD, Frackowiak RSJ (1995b) Statistical parametric maps in functional imaging: a general linear approach. Hum Brain Mapp 2:189-210.

Friston KJ, Holmes A, Poline J-B, Price CJ, Frith CD (1996) Detecting activations in PET and fMRI: levels of inference and power. NeuroImage 4:223-235.

Friston KJ, Holmes AP, Worsley KJ (1999) How many subjects constitute a study? NeuroImage 10:1-5.

Grefkes C, Weiss PH, Zilles K, Fink GR (2002) Crossmodal processing of object features in human anterior intraparietal cortex: an fMRI study implies equivalencies between humans and monkeys. Neuron 35:173-184.

Harada T, Saito DN, Kashikura K, Sato T, Yonekura Y, Honda M, Sadato N (2004) Asymmetrical neural substrates of tactile discrimination in humans: a functional magnetic resonance imaging study. J Neurosci 24:7524-7530.

Holmes AP, Friston KJ (1998) Generalizability, random effects and population inference. NeuroImage 7:S754.

Hopfinger JB, Buonocore MH, Mangun GR (2000) The neural mechanisms of top-down attentional control. Nat Neurosci 3:284-291.

Jones LA, Lederman SJ (2006) Human hand function. New York: Oxford UP.

Kerkhoff G (2001) Spatial hemineglect in humans. Prog Neurobiol 63:1-27. Kesler SR, Haberecht MF, Menon V, Warsofsky IS, Dyer-Friedman J, Neely EK, Reiss AL (2004) Functional neuroanatomy of spatial orientation processing in Turner syndrome. Cereb Cortex 14:174-180.

Lederman SJ (1981) The perception of surface roughness by active and passive touch. Bull Psychon Soc 18:253-255.

Lederman SJ, Klatzky RL (1997) Relative availability of surface and object properties during early haptic processing. J Exp Psychol Hum Percept Perform 23:1680-1707.

Lederman SJ, Taylor MM (1972) Fingertip force, surface geometry and the perception of roughness by active touch. Percept Psychophys 12:401-408.

Lederman SJ, Klatzky RL, Chataway C, Summers C (1990) Visual mediation and the haptic recognition of two dimensional pictures of common objects. Percept Psychophys 47:54-64.

Marmor GS, Zaback LA (1976) Mental rotation by the blind: does mental rotation depend on visual imagery? J Exp Psychol Hum Percept Perform 2:515-521.

Merabet L, Thut G, Murray B, Andrews J, Hsiao S, Pascual-Leone A (2004) Feeling by sight or seeing by touch? Neuron 42:173-179.

Murata A, Gallese V, Luppino G, Kaseda M, Sakata H (2000) Selectivity for the shape, size, and orientation of objects for grasping in neurons of monkey parietal area AIP. J Neurophysiol 83:2580-2601. 
Oldfield RC (1971) The assessment and analysis of handedness: the Edinburgh inventory. Neuropsychologia 9:97-113.

Pietrini P, Furey ML, Ricciardi E, Gobbini MI, Wu WH, Cohen L, Guazzelli M, Haxby JV (2004) Beyond sensory images: object-based representation in the human ventral pathway. Proc Natl Acad Sci USA 101:5658-5663.

Podzebenko K, Egan GF, Watson JD (2002) Widespread dorsal stream activation during a parametric mental rotation task, revealed with functional magnetic resonance imaging. NeuroImage 15:547-558.

Saito DN, Okada T, Morita Y, Yonekura Y, Sadato N (2003) Tactile-visual cross-modal shape matching: a functional MRI study. Brain Res Cogn Brain Res 17:14-25.

Sakata H, Taira M (1994) Parietal control of hand action. Curr Opin Neurobiol 4:847-856.

Sathian K, Zangaladze A, Hoffman JM, Grafton ST (1997) Feeling with the mind's eye. NeuroReport 8:3877-3881.

Talairach J, Tournoux P (1988) A co-planar stereotaxic atlas of a human brain. Stuttgart, Germany: Thieme.

Van Boven RW, Johnson KO (1994a) A psychophysical study of the mechanisms of sensory recovery following nerve injury in humans. Brain 117:149-167.
Van Boven RW, Johnson KO (1994b) The limit of tactile spatial resolution in humans: grating orientation discrimination at the lip, tongue, and finger. Neurology 44:2361-2366.

Van Boven RW, Ingeholm JE, Beauchamp MS, Bikle PC, Ungerleider LG (2005) Tactile form and location processing in the human brain. Proc Natl Acad Sci USA 102:12601-12605.

Vandenberghe R, Dupont P, De Bruyn B, Bormans G, Michiels J, Mortelmans L, Orban GA (1996) The influence of stimulus location on the brain activation pattern in detection and orientation discrimination: a PET study of visual attention. Brain 119:1263-1276.

Worsley KJ, Friston KJ (1995) Analysis of fMRI time-series revisited-again. NeuroImage 2:173-181.

Zangaladze A, Epstein CM, Grafton ST, Sathian K (1999) Involvement of visual cortex in tactile discrimination of orientation. Nature 401:587-590.

Zhang M, Weisser VD, Stilla R, Prather SC, Sathian K (2004) Multisensory cortical processing of object shape and its relation to mental imagery. Cogn Affect Behav Neurosci 4:251-259.

Zhang M, Mariola E, Stilla R, Stoesz M, Mao H, Hu X, Sathian K (2005) Tactile discrimination of grating orientation: fMRI activation patterns. Hum Brain Mapp 25:370-377. 